\title{
Critical peak rebate strategy and application to demand response
}

\author{
Hejun Yang ${ }^{*}$, Xinyu Zhang, Yinghao Ma and Dabo Zhang
}

\begin{abstract}
Time-of-use (TOU) pricing strategy is an important component of demand-side management (DSM), but the cost of supplying power during critical peak periods remains high under TOU prices. This affects power system reliability. In addition, TOU prices are usually applicable to medium- and long-term load control but cannot effectively regulate short-term loads. Therefore, this paper proposes an optimization method for TOU pricing and changes the electricity consumption patterns during the critical peak periods through a critical peak rebate (CPR). This reduces generation costs and improves power system reliability. An optimization model for peak-flat-valley (PFV) period partition is established based on fuzzy clustering and an enumeration iterative technique. A TOU pricing optimization model including grid-side and customer-side benefits is then proposed, and a simulated annealing particle swarm optimization (SAPSO) algorithm is used to solve the problem. Finally, a CPR decision model is developed to further reduce critical peak loads. The effectiveness of the proposed model and algorithm is illustrated through different case studies of the Roy Billinton Test System (RBTS).
\end{abstract}

Keywords: Demand side management (DSM), TOU price optimization, Critical peak rebate, Reliability analysis

\section{Introduction}

Demand response refers to the demand side management mode in which users transfer or reduce loads in response to electricity prices or incentive signals. Time-of-use (TOU) pricing is an important part of demand response strategy [1-3], which can not only delay the need for grid investment, but also improve power system stability, and thus has been widely used in the electricity market [4-6]. However, as discussed in [7], users are not well prepared to respond to time-varying prices, and therefore TOU pricing is usually only applied to medium and long-term load regulation. Thus, even if peak-flat-valley (PFV) TOU pricing is implemented, load in the critical peak period (i.e., critical peak load) will still be high [8]. The presence of critical peak load not only reduces the utilization rate of power equipment, but also affects the safe and reliable operation of the power system. Therefore, it is necessary

\footnotetext{
*Correspondence: cquyhj@126.com

Anhui Provincial Laboratory of Renewable Energy Utilization and Energy

Saving (Hefei University of Technology), Hefei 230009, Anhui Province,
} China to combine TOU pricing with short-term critical peak load regulation to improve system reliability and the economy of system operation.

PFV period partition is an important part of TOU electricity price research. Several studies in [9-11] derive period partition results directly from experience, while ignoring the difference of period partition in different cases. The K-means clustering algorithm is used in [12] to obtain the best period partition results, though the randomness of initial values can cause nonunique partial results. In [13], a novel approach is proposed to efficiently reveal the latent cluster structure of multi-view data for clustering, while [14] introduces a classifier design method based on a modification of traditional fuzzy clustering. An evolving fuzzy clustering approach is developed in [15], which uses a fuzzy membership concept to break down clustering in epochs instead of running the clustering of all data at once. To integrate more comprehensive information from different views, reference [16] develops a fuzzy clustering model using a low-rank tensor to address 
the multi-view data clustering problem. However, the algorithms in [13-16] are complex to implement and have difficulty in meeting the fuzzy judgment criteria of period partition. An enumeration iteration method is used in [17] to partition periods of each month. However, it is sensitive to outliers and has no constraints on the length of the period. Since the length of the period will affect user electricity consumption habits, it needs to be limited.

TOU electricity prices are usually determined according to multiple objectives and obtained through optimization algorithms. In [17], a TOU price optimization model considering the benefits of the grid side is proposed, while [18] develops a consumer satisfaction indicator to measure the quality of the user experience. In [19], an optimal TOU electricity pricing model is investigated and the particle swarm optimization (PSO) algorithm is adopted to solve the proposed optimization problem. However, although the PSO algorithm has a high convergence speed, it is easy for it to converge to a local optimal solution. The simulated annealing (SA) algorithm introduced in [20] has high global convergence, but takes a long time to converge.

Critical peak pricing $(\mathrm{CPP})$ is a pricing mechanism proposed for the critical peak load, and it can guide users to reduce or transfer their critical peak loads [21]. At present, most CPP-related research is based on a fully open electricity market environment [22]. Reference [23] introduces the design and implementation of CPP among types of electricity users, while the study presented in [24] develops a critical peak rebate (CPR) strategy in the CPP mechanism and analyzes the load adjustment effects of TOU price and CPP strategy, respectively. In [8], a TOU pricing with incentive strategy is developed without considering the incentive cost and the impact of incentives on other periods.

This paper mainly studies the load regulation effect on TOU electricity pricing and CPR strategy, and analyzes the reliability of the regulation results. A period partition method based on fuzzy clustering and enumeration iteration is proposed first to obtain the optimal period partition. It uses an "exponential similarity coefficient" instead of "Euclidean distance" to dilute the influence of outliers on moving variables. To meet the interests of both grid and user, a multi-objective PFV TOU electricity price optimization model is then established. A simulated annealing particle swarm optimization (SAPSO) algorithm is then proposed with high convergence speed and global convergence for the optimal TOU prices. A critical peak load regulation model based on CPR strategy is constructed to combine critical peak load regulation with TOU pricing, where the critical peak rebate in the model is derived from users' power shortage cost $[25,26]$. Finally, the Roy Billinton Test System (RBTS) system is used in case studies to prove the correctness and effectiveness of the proposed model and algorithm.

The distinctive features and innovative contributions of this paper are as follows:

1. Based on fuzzy clustering theory, this paper proposes a PFV period partition model, which can comprehensively analyze the load data of several typical days and add length constraints to the periods. An iterative algorithm is then proposed to find the optimal period partition scheme. In addition, to reduce the influence of outliers on the position of moving variables, an exponential similarity formula is introduced to characterize the similarity of each time to different periods.

2. Considering the interests of both grid and demand sides, an optimization model of TOU pricing is proposed and is solved using the SAPSO algorithm, which is developed based on the PSO and SA algorithms to ensure high convergence speed and global convergence.

3. Within the context of TOU pricing, a critical peak load adjustment model based on CPR strategy is proposed to further reduce peak load. In this model, the critical peak load reduction is determined by an elastic coefficient adjustment strategy and the principle of proportional distribution is implemented by signing agreements with users.

4. A CPR decision-making model based on power shortage cost is proposed to compensate users' participation in the CPR strategy and to further improve the stability of the power system.

\section{Optimal period partition iteration method based on fuzzy clustering}

According to TOU electricity pricing theory, a typical day is divided into a few PFV periods [1]. Thus this section establishes a period partition model based on fuzzy clustering theory and presents a period partition algorithm based on an enumeration iterative algorithm.

\subsection{Period partition optimization model}

Users' load characteristics in a single quarter are similar, so this section divides PFV periods by analyzing the load data in a quarter. A typical day in each month is selected, and the load data of three typical days in each quarter are grouped to divide into PFV periods. From 
fuzzy clustering theory, three fuzzy judgment criteria are designed, i.e.:

1. The probability of the lowest point on the load curve being in the valley period is $100 \%$, and the probability of the highest point being in the valley period is $0 \%$.

2. The probability of the highest point on the load curve being in the peak period is $100 \%$, and the probability of the lowest point being in the peak period is $0 \%$.

3. The possibilities of other points of the load curve belonging to different periods are determined by the semi trapezoid membership.

If the load at the time $i$ of the typical day of the $j$ th month in the quarter is expressed as $q_{i j}$, where $j=1,2,3$ and $i=1$, $2, \ldots, 24$, and $q_{\text {minj }}$ and $q_{\text {maxj }}$ represent the respective minimum and maximum loads during a typical day of the $j$ th month, the range normalization of $q_{i j}$ is expressed according to semi trapezoid peak membership degree as:

$$
U_{i j}=\frac{q_{i j}-q_{\text {minj }}}{q_{\text {maxj }}-q_{\text {minj }}}
$$

where $U_{i j}$ represents the peak membership degree of time $i$ in the typical day of the $j$ th month.

The peak membership degree $U_{i}$ which represents the probability of time $i$ being in the peak period is given as:

$$
U_{i}=\sqrt{\frac{\sum_{j=1}^{3} U_{i j}^{2}}{3}}
$$

According to $U_{i}$ values from small to large, all times are sorted and boundaries between different periods are determined jointly by peak and valley membership degrees. These cannot be obtained by $U_{i}$ alone. Therefore, this paper introduces moving variables and reference points of peak membership. $(0,0,0)$ and $(1,1,1)$ are the reference points of the minimum and maximum peak membership, respectively, while the moving variables are the points on the shortest distance between $(0,0,0)$ and $(1,1,1)$. The boundaries are determined by calculating the maximum similarity between the peak membership degrees at each moment and the corresponding moving variables.

$m_{p}, m_{f}$ and $m_{v}$ are defined as the moving variables of membership degree in PFV periods, and are uniformly expressed as $m_{s=\{p, f v\}\}}$. To reduce the influence of outliers on the position of the moving variables, the similarity $r_{i s}$ between the time $i$ and the moving variables $s$ can be obtained by:

$$
r_{i s}=\frac{1}{3} \sum_{j=1}^{3} e^{-\frac{3}{4}\left(U_{i j}-m_{s}\right)^{2}}
$$

In this section, length constraints are added to period partition results, and the PFV period partition model can be expressed as:

$$
\begin{aligned}
& \max \left\{\sum_{s=\{p, f, v\}} \sum_{i \in G_{s}} r_{i s}\right\} \\
& \left\{\begin{array}{l}
m_{p}-m_{f}>0 \\
m_{f}-m_{v}>0 \\
0 \leq m_{s=\{p, f, v\}} \leq 1
\end{array}\right. \\
& \left\{\begin{array}{l}
l_{\min } \leq \operatorname{card}\left(G_{s=\{p, f, v\}}\right) \leq l_{\max } \\
\sum_{s=\{p, f, v\}} \operatorname{card}\left(G_{s}\right)=24 \\
2 l_{\min }+l_{\max } \leq 24 \\
2 l_{\max }+l_{\min } \geq 24
\end{array}\right.
\end{aligned}
$$

where $G_{s}$ represents the time set of period $s$, and $\operatorname{card}\left(G_{s}\right)$ represents the number of times in the period $s . l_{\min }$ and $l_{\max }$ represent the minimum and maximum of the period length, respectively.

\subsection{Period partition iterative algorithm}

The three moving variables are considered to move in equal steps, and the objective function is calculated step by step. The period partition result is selected as the optimal result when the objective function is the largest. The calculation process of the optimal result is as follows:

Step 1 Set the moving step size of $m_{s=\{p, f, v\}}$ as:

$$
\Delta m=\frac{1}{N}
$$

where $N$ is the maximum number of steps that the moving variable moves.

Step 2 Initialize moving variables. Since $m_{s} \in[0,1]$, with the constraint conditions shown in (4b), $m_{s=\{p, f, v\}}$ is initialized as:

$$
\left\{\begin{array}{l}
m_{v}=0 \\
m_{f}=m_{v}+\Delta m \\
m_{p}=m_{f}+\Delta m
\end{array}\right.
$$

Step 3 Determine the period partition. The 24 times are sorted in positive order according to the values of $U_{i}$, and the sequence corresponding to time $i$ is set as $L_{i}$. In the light of (4c), it is considered that when $L_{i} \in\left[1, l_{\text {min }}\right]$, the probability $U_{i}$ of $i$ is small, so $i \in G_{v}$. When $L_{i} \in\left[25-l_{\text {min }}\right.$, 24], $U_{i}$ is large, so $i \in G_{p} . l_{\min }$ times are then added to the largest $r_{i f}$ from remaining times to $G_{f}$, and the maximum 
$r_{i s=\{p, f v\}}$ of each remaining time is calculated and classified into the corresponding set $G_{s}$.

Step 4 Calculate the value of the objective function. If $\operatorname{card}\left(G_{s=\{p, f, v\}}\right)>l_{\max }$, the objective function value of this iteration is 0; otherwise, it is calculated according to (4a).

Step 5 Determine the optimal results. If the current value of the objective function is greater than the optimal one, the current value is used as the optimal value and the period partition results are saved as the optimal results.

Step 6 Determine if the iteration is terminated. If $m_{p}=1$, $m_{f}=1-\Delta m$ and $m_{v}=1-2 \Delta m$, terminate the iteration and go to Step 7. Otherwise, find the first $m_{s=\{p, f, v\}}$ that does not satisfy the stopping criteria in descending order, set $m_{s}=m_{s}+\Delta m$ and initialize the boundary variables greater than $m_{s}$ according to (6), and then return to Step 3.

Step 7 Output optimal period partition results.

\section{Optimization model of PFV TOU electricity price}

In this section, the objective functions of the grid-side and user-side are integrated to establish a TOU electricity price optimization model. The SAPSO algorithm is then proposed to obtain the optimal electricity prices.

\subsection{Grid side objective functions}

The objective functions of the grid-side are to minimize the peak load and the peak-to-valley load difference, as:

$$
\left\{\begin{array}{l}
F_{1}\left(p_{p}, p_{f}, p_{v}\right)=\min \left\{\max _{1 \leq i \leq 24}\left[q_{i}^{\prime}\left(p_{p}, p_{f}, p_{v}\right)\right]\right\} \\
F_{2}\left(p_{p}, p_{f}, p_{v}\right)=\min \left\{\begin{array}{l}
\max _{1 \leq i \leq 24}\left[q_{i}^{\prime}\left(p_{p}, p_{f}, p_{v}\right)\right] \\
-\min _{1 \leq i \leq 24}\left[q_{i}^{\prime}\left(p_{p}, p_{f}, p_{v}\right)\right]
\end{array}\right\}
\end{array}\right.
$$

where $F_{1}(\cdot)$ and $F_{2}(\cdot)$ are the objective functions, $p_{p}, p_{f}$, and $p_{v}$ are decision variables, and $q_{i}^{\prime}(\cdot)$ denotes the optimized power at time $i$.

\subsection{User side objective functions}

The maximum power consumption similarity and the maximum user satisfaction are taken as objective functions:

$$
\left\{\begin{array}{l}
F_{3}\left(p_{p}, p_{f}, p_{v}\right)=\max (K) \\
F_{4}\left(p_{p}, p_{f}, p_{v}\right)=\max (S)
\end{array}\right.
$$

The power consumption similarity $K$ (Spearman similarity equation) and the user satisfaction $S$ are:

$$
K=\frac{\sum_{i=1}^{24}\left(q_{i}-\bar{q}\right)\left(q_{i}^{\prime}-\overline{q^{\prime}}\right)}{\sqrt{\sum_{i=1}^{24}\left(q_{i}-\bar{q}\right)^{2}} \sqrt{\sum_{i=1}^{24}\left(q_{i}^{\prime}-\overline{q^{\prime}}\right)^{2}}}
$$

$$
S=\frac{\sum_{i=1}^{24} q_{i} p_{0}-\sum_{i=1}^{24} q_{i}^{\prime} p_{i}}{\sum_{i=1}^{24} q_{i} p_{0}}
$$

where $q_{i}$ and $q_{i}^{\prime}$ represent the loads at the time $i$ before and after optimization, respectively, whereas $\bar{q}$ and $\bar{q}^{\prime} q$ and ${ }^{-} q^{\prime}$ are the respective average loads before and after optimization. $p_{0}$ is the initial price and $p_{i}$ represents the TOU price at time $i$.

\subsection{Constraint functions of PFV TOU Pricing}

1. Users' benefit After implementing the TOU price, the electricity expense of users will not increase, i.e.:

$$
g_{1}\left(p_{p}, p_{f}, p_{\nu}\right)=\sum_{s=\{p, f, v\}}\left(Q_{s} p_{0}\right)-\sum_{s=\{p, f, v\}}\left(Q_{s}^{\prime} p_{s}\right) \geq 0
$$

where $g_{1}(\cdot)$ is the constraint function. $Q_{s}$ and $Q_{s}^{\prime}$ are the loads in period $s$ before and after optimization, whereas $p_{0}$ and $p_{s}$ are the electricity prices in period $s$ before and after optimization, respectively.

2. Benefit to the power supplier the stability of the power system will be improved considering the TOU price, and the power supply cost will be reduced. The total benefit to the power supplier will not be reduced after the TOU price, i.e.:

$$
g_{2}\left(p_{p}, p_{f}, p_{v}\right)=\sum_{s=\{p, f, v\}}\left(Q_{s}^{\prime} p_{s}\right)-(1-\lambda) \sum_{s=\{p, f, v\}}\left(Q_{s} p_{0}\right) \geq 0
$$

where $\lambda$ is the benefit coefficient [27].

3. Electricity rate To prevent the peak and valley periods from being inverted, it is necessary to restrict the price relationship of each period, as:

$$
\left\{\begin{array}{l}
g_{3}\left(p_{p}, p_{f}, p_{v}\right)=p_{p}-p_{f}>0 \\
g_{4}\left(p_{p}, p_{f}, p_{v}\right)=p_{f}-p_{v}>0
\end{array}\right.
$$

4. Marginal cost price The price in the valley period shall be higher than the marginal cost price in the valley period, i.e.:

$$
g_{5}\left(p_{p}, p_{f}, p_{v}\right)=p_{v}-p_{c}>0
$$

where $p_{c}$ is the marginal cost price.

5. Electricity consumption similarity To make the optimal load strategy similar to the users' power consumption habits, it is necessary to ensure that the 
load distributions before and after optimization are consistent, i.e.:

$$
\left\{\begin{array}{l}
g_{6}\left(p_{p}, p_{f}, p_{v}\right)=\eta \min \left(Q_{p}\right)-\max \left(Q_{f}\right) \geq 0 \\
g_{7}\left(p_{p}, p_{f}, p_{v}\right)=\eta \min \left(Q_{f}\right)-\max \left(Q_{v}\right) \geq 0
\end{array}\right.
$$

where $\eta$ is the adjustment coefficient.

6. Total electricity consumption: The total daily load should be guaranteed to change within a certain range before and after the TOU price as:

$$
\left\{\begin{array}{l}
g_{8}\left(p_{p}, p_{f}, p_{v}\right)=\frac{\sum_{s=\{p, f, v\}} Q_{s}}{\sum_{s=\{p, f, v\}} Q_{s}^{\prime}}-0.9 \geq 0 \\
g_{9}\left(p_{p}, p_{f}, p_{v}\right)=1.1-\frac{\sum_{s=\{p, f, v\}} Q_{s}}{\sum_{s=\{p, f, v\}} Q_{s}^{\prime}} \geq 0
\end{array}\right.
$$

\subsection{Single-objective fitness function of TOU optimization}

This section transforms the multi-objective function into a single objective function by proportion coefficients and the penalty function $J(\boldsymbol{P})$ related to the constraints as:

$$
F(\mathbf{P})=\beta_{1} F_{1}(\mathbf{P})+\beta_{2} F_{2}(\mathbf{P})+\beta_{3} F_{3}(\mathbf{P})+\beta_{4} F_{4}(\mathbf{P})+J(\mathbf{P})
$$

where $\beta_{i}$ is the ratio coefficient of objective function $F_{i}(\cdot)$ and can be used to represent different user types. $\boldsymbol{P}=\left(p_{p}\right.$, $\left.p_{f}, p_{v}\right)$ and $J(\boldsymbol{P})$ is the penalty function from [28].

\subsection{Calculation method of hourly load}

The price elasticity coefficient of demand can show the sensitivity of user demand to price change. This section defines $e_{i j}(i, j=p, f, v)$ as the price elasticity coefficient, which represents the impact of the price change in period $j$ on the load in period $i$. The effect of the price change on demand is given as:

$$
\left[\begin{array}{c}
\frac{\Delta Q_{p}}{Q_{p}} \\
\frac{\Delta Q_{f}}{Q_{f}} \\
\frac{\Delta Q_{v}}{Q_{v}}
\end{array}\right]=\left[\begin{array}{ccc}
e_{p p} & e_{p f} & e_{p v} \\
e_{f p} & e_{f f} & e_{f v} \\
e_{v p} & e_{v f} & e_{v v}
\end{array}\right]\left[\begin{array}{c}
\frac{\Delta p_{p}}{p_{0}} \\
\frac{\Delta p_{f}}{p_{0}} \\
\frac{\Delta p_{v}}{p_{0}}
\end{array}\right]
$$

where $Q_{p}, Q_{f}$ and $Q_{v}$ denote the power consumption in peak, flat and valley periods at the initial price, respectively, whereas $\Delta Q_{p}, \Delta Q_{f}$ and $\Delta Q_{v}$ are the respective load changes in different periods after the TOU price. $\Delta p_{p}, \Delta p_{f}$ and $\Delta p_{v}$ are the respective changes of price in different periods considering the TOU price.

From (16), the load change at each time after the TOU price can be further calculated as:

$$
\Delta q_{i}=\frac{q_{i}}{k_{l}} \sum_{l=1}^{24} e_{i l} \frac{p_{l}-p_{0}}{p_{0}}
$$

where $i$ and $l$ are times, and $\Delta q_{i}$ and $q_{i}$ are the load change and initial load at the time $i$, respectively. $e_{i l}$ is the elasticity coefficient corresponding to the periods of time $i$ and time $l . k_{l}$ is the number of times in the period $l$ and $p_{l}$ is the electricity price at the time $l$ under the TOU price.

The load $q_{i}^{\prime}$ at the time $i$ under the TOU price is:

$$
q_{i}^{\prime}=q_{i}+\Delta q
$$

\subsection{Optimization method of PFV TOU price based on the SAPSO algorithm}

This section combines the PSO algorithm with the SA algorithm and proposes a SAPSO algorithm which has high convergence speed and global convergence, to obtain the optimal TOU electricity prices.

In this section, the new variables are defined as follows: $M$ is the iteration number, $i$ denotes the $i$ th particle, and $j$ denotes the $j$ th dimension. Each particle has three dimensions, $j=1$ for the peak period, $j=2$ for the flat period, and $j=3$ for the valley period. Each particle has position and velocity variables, which are continuously updated during iterations. $X_{i j}{ }^{k}$ and $V_{i j}{ }^{k}$ are the position and velocity variables of the $j$ th dimension of the $i$ th particle in the $k$ th iteration. $X_{\max }$ and $X_{\min }$ denote the upper and lower limits of the position variable, while $V_{\max }$ and $V_{\text {min }}$ represent the upper and lower limits of the velocity variable. $B_{i}$ and $B^{\prime}$ are the local and global optimal fitness variables of particle $i . b_{i j}$ is the optimal position variable of the $j$ th dimension of the $i$ th particle, while $b_{g j}$ is the optimal position variable of the $j$ th dimension of all particles. $T_{0}$ is the initial temperature, $T$ is the temperature variable and $D$ is the decay rate of temperature.

The optimization steps of TOU pricing based on the SAPSO algorithm are as follows:

Step 1 Initialize position variables, velocity variables, and temperature variables.

- Select $2 \times 3 \times M$ random numbers $R_{i j}(i=1,2, \ldots, M$; $j=1,2,3)$, which are uniformly distributed in $[0,1]$, and set $k=0$.

- Initialize the position variable $X_{i j}{ }^{0}$, velocity variable $V_{i j}{ }^{0}$ of each particle, and the temperature variable $T$ as:

$$
\left\{\begin{array}{c}
X_{i j}^{0}=R_{i j}\left(X_{\max }-X_{\min }\right)+X_{\min } \\
V_{i j}^{0}=R_{i j}\left(V_{\max }-V_{\min }\right)+V_{\min } \\
T=T_{0}
\end{array}\right.
$$

Step 2 Initialize local and global optimization variables. 
- Calculate the fitness $F\left[X_{i}^{0}\right]$ of all particles and assign $F\left[X_{i}^{0}\right]$ to the local optimal fitness variable $B_{i}$. It is assumed that the local optimal location variable $b_{i j}$ is the location variable $X_{i j}{ }^{0}$.

- Compare the local optimal fitness variable $B_{i}$ of all particles, assign the minimum value to the global optimal fitness variable $B^{\prime}$, and assign its location variable to the global optimal location variable $b_{g j}$.

- Let $k=k+1$.

Step 3 Update velocity and position variables.

- Select $3 \times M$ random numbers $H_{i j}$, which are uniformly distributed in $(-1,1)$.

- Update the velocity variable $V_{i j}{ }^{0}$ and position variable $X_{i j}{ }^{0}$ of all particles as:

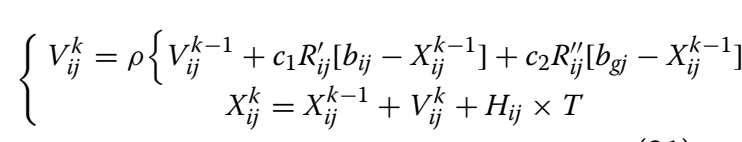

where $c_{1}$ and $c_{2}$ are constants, $R^{\prime}{ }_{i j} \sim \mathrm{U}(0,1)$ and $R^{\prime}{ }_{i j} \sim \mathrm{U}(0,1)$ are two independent random variables, and $\rho$ is the contraction factor, which can be calculated by:

$$
\rho=\frac{2}{\left|2-\left(c_{1}+c_{2}\right)-\sqrt{\left(c_{1}+c_{2}\right)^{2}-4\left(c_{1}+c_{2}\right)}\right|}
$$

- Limit the position variable according to the upper and lower limits as:

$$
X_{i j}^{k}=\left\{\begin{array}{lc}
X_{\max } & X_{\max }<X_{i j}^{k} \\
X_{i j}^{k} & X_{\min }<X_{i j}^{k} \leq X_{\max } \\
X_{\min } & X_{i j}^{k} \leq X_{\min }
\end{array}\right.
$$

Step 4 Calculate the fitness of all particles.

- Calculate the fitness $F\left[X_{i}^{k}\right]$ of the $i$ th particle in the $k$ th iteration and compare it with the fitness $F\left[X_{i}^{k-1}\right]$ in the previous iteration. If $F\left[X_{i}^{k}\right] \leq F\left[X_{i}^{k-1}\right]$, the $i$ th particle remains the position variable $X_{i j k^{k}}^{k}$ Otherwise, the particle has the probability of exp $\frac{F\left[X_{i}^{k-1}\right] \leq F\left[X_{i}^{k}\right]}{T}$ to keep the position variable unchanged, and the probability of $1-\exp \frac{F\left[X_{i}^{k-1}\right] \leq F\left[X_{i}^{k}\right]}{T}$ to change the position variable as:

$$
X_{i j}^{k}=X_{i j}^{k-1}+V_{i j}^{k}
$$

- If the position variable of particle $i$ changes, recalculate the fitness variable $F\left[X_{i}^{k}\right]$.
Step 5 Update the local and global optimization variables of all particles.

- Compare the fitness variable $F\left[X_{i}^{k}\right]$ of the $i$ th particles in the $k$ th iteration with the local optimal fitness variable $B_{i}$. If $F\left[X_{i}^{k}\right]<B_{i}, B_{i}=F\left[X_{i}^{k}\right]$, and $b_{i j}=X_{i j}{ }^{k}$; otherwise, keep $B_{i}$ and $b_{i j}$ unchanged.

- Compare the minimum local optimal fitness $\min \left(B_{i}\right)$ of all particles with the global optimal fitness variable $B^{\prime}$. If $\min \left(B_{i}\right)<B^{\prime}$, then $B^{\prime}=\min \left(B_{i}\right)$, and $b_{g j}=b_{i j}$; otherwise, keep $B^{\prime}$ and $b_{g j}$ unchanged.

Step 6 Update the temperature variable as follows:

$$
T=T \times D
$$

Step 7 Termination criterion of iteration.

Check whether the fitness converges to the rated value. Iff so, output the global optimal fitness variable $B^{\prime}$, the global optimal location variable $b_{g j}(j=1,2,3)$, and the optimized load at each time; otherwise, let $k=k+1$ and return to step 3.

\section{Critical peak load regulation based on the CPR strategy}

This section further divides the critical peak period and regulates the critical peak load. The third-order price elasticity matrix in the previous section cannot directly reflect the elastic relationship between the critical peak period and other periods. Therefore, based on the CPR strategy, a critical peak load regulation model is presented. This signs contracts with users to determine the load to be directly reduced in the critical peak period and provides users with the critical peak rebate for load reduction. In this paper, the scheme of implementing CPR under TOU pricing is set as a TC strategy.

\subsection{Critical peak period partition}

In this section, the daily critical peak loads are adjusted separately. Because the daily load distribution may differ greatly from that of the quarterly load, the periods are divided again according to the daily load under TOU pricing, and the steps are as follows:

Step 1 Input the daily load data under TOU pricing, and divide PFV periods according to the TOU period partition method. Because the daily load is analyzed instead of the quarterly load, the dimension of variable $q_{i j}$ is 1 , and $U_{i}=\frac{q_{\mathrm{i}}-q_{\min }}{q_{\max }-q_{\min }}$.

Step 2 Save the period partition results in step 1 and the value of the peak period moving variable $m_{p}$. The peak membership degree $U_{i}$ of each time in the peak period is compared with $m_{p}$, and if $U_{i} \geq m_{p}$, time $i$ belongs to the 
critical peak period; otherwise, time $i$ belongs to the peak period.

Step 3 Output period partition results.

\subsection{Load adjustment strategy based on proportional allocation principle}

The critical peak load adjustment strategy is based on the premise that the period partition results before and after the CPR strategy are consistent. The load adjustment process is as follows:

- Calculate the load to be reduced in the critical peak period.

- Calculate the load increase in other periods according to the historical data and elastic coefficients.

- Predict the load of each time after CPR strategy according to the proportional apportionment principle.

Set $Q_{s=\{p, f, v\}}^{\prime}$ as the total load in period $s$ under TOU pricing. $Q_{p}^{\prime}$ is the total load in the peak period, and $p 1$ and $c p$ the peak period and the critical peak period after the critical peak period partition, respectively. Then $Q_{p}^{\prime}=Q_{p 1}^{\prime}+Q_{c p}^{\prime}$, where $Q_{p 1}^{\prime}$ is the load of the peak period and $Q_{c p}^{\prime}$ is the load of the critical peak period. Let $\theta_{c p}$ be the critical peak-peak load transfer rate, $\theta_{c f}$ the critical peak-flat load transfer rate, $\theta_{c v}$ the critical peak-valley load transfer rate, then $\theta_{c p}+\theta_{c f}+\theta_{c v}=1$. $Q^{\prime}{ }_{s=\{c p, p l, f, v\}}$ denotes the load of each period after CPR strategy, and $\Delta Q_{c p}$ denotes the load reduction of the critical peak period under CPR. Assuming that the total load of the day remains unchanged after CPR strategy, the load of each period under CPR is:

$$
Q_{s=\{c, p 1, f, v\}}^{\prime \prime}=\left\{\begin{array}{cc}
Q_{v}^{\prime}+\theta_{c v} \Delta Q_{c p} & (s=v) \\
Q_{f}^{\prime}+\theta_{c f} \Delta Q_{c p} & (s=f) \\
Q_{p 1}^{\prime}+\theta_{c p} \Delta Q_{c p} & (s=p 1) \\
Q_{c p}^{\prime}-\Delta Q_{c p} & (s=c p)
\end{array}\right.
$$

Then the load at each time, according to the proportional apportionment principle, is calculated by:

$$
q_{i}^{\prime \prime}=\frac{q_{i}^{\prime}}{Q_{s=\{c p, p 1, f, v\}}^{\prime}} Q_{s=\{c p, p 1, f, v\}}^{\prime \prime}
$$

where $s$ is the period of time $i, q^{\prime}{ }_{i}$ and $q^{\prime \prime}{ }_{i}$ denote the load of time $i$ before and after the CPR strategy, and $Q_{s=\{c, p l l, v\}}^{\prime}$ and $Q_{s=\{c, p l, f, v\}}$ represent the load of period $s$ before and after the CPR strategy, respectively.

In order to maximize the value of $\Delta Q_{c p}$ without changing the period partition, $\Delta Q_{c p}$ can be obtained by:

$$
\left\{\begin{array}{c}
q_{(c p) \min }^{\prime}-\Delta q=q_{(p) \max }^{\prime \prime} \\
\gamma=\frac{\Delta q}{q_{(c p) \min }^{\prime}} \\
\Delta Q_{c p}=\gamma Q_{c p}^{\prime}
\end{array}\right.
$$

where $q_{(c p) \text { min }}^{\prime}$ is the minimum load in the critical peak period before the CPR strategy, $\Delta q$ is the corresponding load reduction after the CPR strategy, and $q_{(p) \text { max }}^{\prime}$ is the maximum load in the peak period under CPR. $\gamma$ is the load reduction rate in the critical peak period under CPR.

\subsection{Calculation of load transfer rate}

This section uses the price elasticity matrix and the load data at each time under TOU pricing to find the load transfer rate. The CPR and the peak price are assumed to have the same effect on the flat load and valley load, i.e.:

$$
\frac{\theta_{c f}}{\theta_{c v}}=\frac{e_{p f} Q_{f}^{\prime}}{e_{p v} Q_{v}^{\prime}}
$$

Let $\theta_{c p}: \theta_{c \dot{j}} \theta_{c v}=a_{1}: a_{2}: a_{3} . a_{2}$ and $a_{3}$ can be expressed as:

$$
\left\{\begin{array}{l}
a_{2}=e_{p f} Q_{f}^{\prime} \\
a_{3}=e_{p v} Q_{v}^{\prime}
\end{array}\right.
$$

$a_{1}$ can be approximately obtained from the relationship between load changes in different periods under TOU pricing as:

$$
a_{1}=\frac{Q_{p 1}^{\prime}}{2 Q_{p}^{\prime}}\left(\frac{e_{f p} Q_{p}^{\prime}}{e_{f v} Q_{v}^{\prime}} a_{3}+\frac{e_{v p} Q_{p}^{\prime}}{e_{v f} Q_{f}^{\prime}} a_{2}\right)
$$

Load transfer rates are calculated by:

$$
\left\{\begin{array}{l}
\theta_{c p}=\frac{a_{1}}{a_{1}+a_{2}+a_{3}} \\
\theta_{c f}=\frac{a_{2}}{a_{1}+a_{2}+a_{3}} \\
\theta_{c v}=\frac{a_{3}}{a_{1}+a_{2}+a_{3}}
\end{array}\right.
$$

\subsection{CPR decision model}

The power shortage cost $C\left(q_{i}\right)$ in [26] has a quadratic relationship with load reduction $q_{i}$ at time $i$ as:

$$
C\left(q_{i}\right)=k_{1} q_{i}^{2}+k_{2} q_{i}-k_{2} q_{i} \tau_{i}
$$

where $C\left(q_{i}\right)$ is the cost of reducing the load $q_{i}, \tau_{i}$ is the user type parameter, and $k_{1}$ and $k_{2}$ are constant coefficients. In this paper, the data in [26] is used, i.e., $k_{1}=0.5$, $k_{2}=1$. The benefit of power reduction is:

$$
C^{\prime}\left(q_{i}\right)=\left(p_{i}+a_{i}\right) q_{i}
$$

where $C^{\prime}\left(q_{i}\right)$ denotes the benefit of load reduction $q_{i}$, and $C^{\prime}\left(q_{i}\right)=C\left(q_{i}\right) \cdot p_{i}$ is the TOU price at time $i$, and $a_{i}$ is the rebate corresponding to time $i$. The relationship between $a_{i}$ and $q_{i}$ is: 


$$
\left\{\begin{array}{l}
a_{i}+p_{i}=k_{1} q_{i}+k_{2}\left(1-\tau_{i}\right) q_{i} \neq 0 \\
a_{i}=0 q_{i} \rightarrow 0
\end{array}\right.
$$

From the above equations, when $q_{i} \neq 0, a_{i}=k_{1} q_{i}$, and the daily rebate $A$ during the critical peak period can be obtained as:

$$
\sum_{i \in G_{c p}} q_{i} A=\sum_{i \in G_{c p}} q_{i} a_{i}
$$

where $G_{c p}$ represents the set of critical peak times.

\section{Case study}

\subsection{Basic data}

In this study, to simplify the calculation, the maximum peak load day in each month is selected as the typical day. The minimum and maximum lengths of period $l_{\text {min }}$ and $l_{\text {max }}$ are 6 and 10, respectively. The ratio coefficients are $\beta_{1}=\beta_{2}=0.5, \beta_{3}=\beta_{4}=-0.3$, the benefit coefficient $\lambda$ is 0.062 , the marginal cost $p_{c}$ is $0.35 \mathrm{RMB}$, the adjustment coefficient $\eta$ is 1.2 , the initial price $p_{0}$ is $0.65 \mathrm{RMB}$, and the elasticity coefficients are set according to [17]. The load data and reliability test data are provided by the RBTS system [29, 30], and the peak load is $185 \mathrm{MW}$. For statistical purposes, it considers 30 days in a month.

\subsection{Optimal peak-flat-valley period partition}

According to the period partition optimization model, the optimal period partition results of four quarters are obtained. Figure 1 shows the initial load of the first two quarters with different periods distinguished by color markings. In the first quarter, the peak periods are 9:00-13:00 and 17:00-20:00, the flat periods are 8:00,

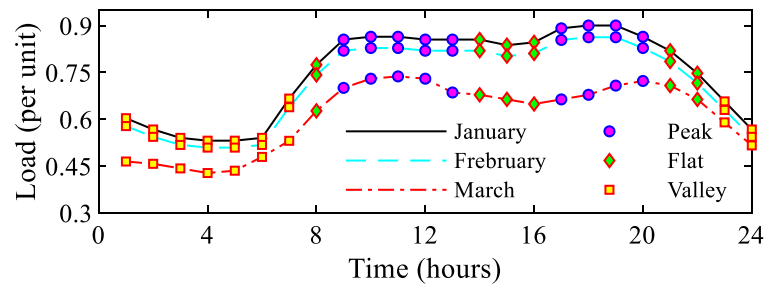

(a)

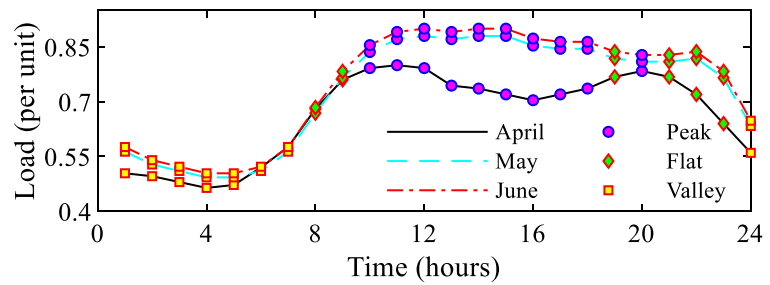

(b)

Fig. 1 Period partitioning for: a first quarter $\mathbf{b}$ second quarter

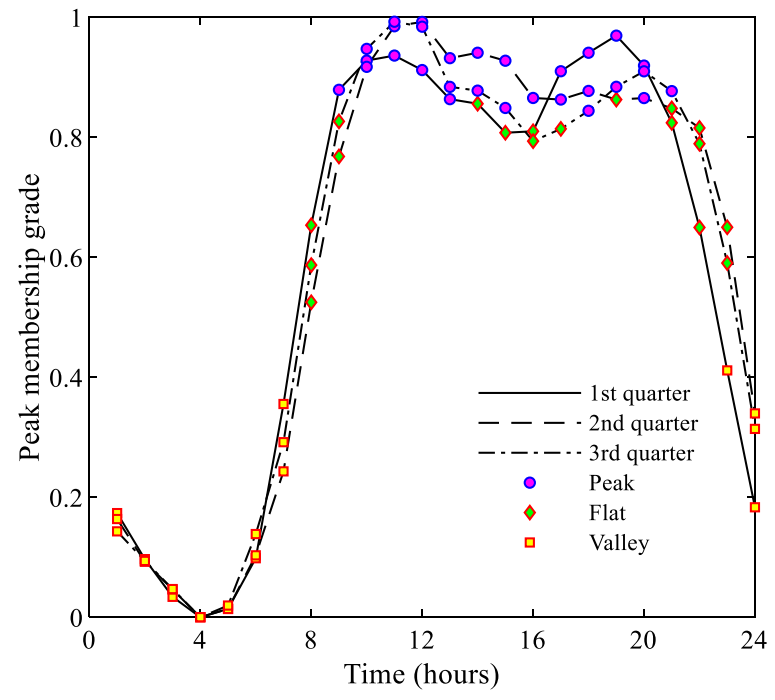

Fig. 2 Peak membership of each time

14:00-16:00 and 21:00-22:00, while the valley periods are 1:00-7:00 and 23:00-24:00.

Since the load data of the fourth quarter is similar to that of the first quarter, the first three quarters are thus taken as an example to show the peak membership degree of each time in different quarters. The period partition results of the three quarters are shown in Fig. 2.

Taking the first quarter as an example, the value of the objective function and the length of the iteration time are analyzed, as shown in Fig. 3. Figure 3a shows that when the number of iterations $N=48$, the function value tends to be static, and it takes $0.262 \mathrm{~s}$ to complete period

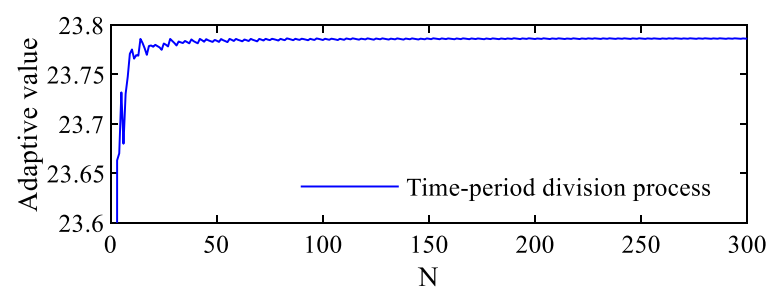

(a)

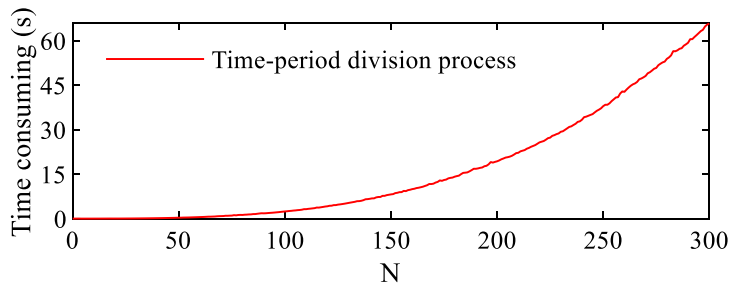

(b)

Fig. 3 Iterative process of period partition 
partition as shown in Fig. 3b. It can be concluded that the proposed period partition method is efficient.

\subsection{Analysis of SAPSO algorithm}

The influence of randomness in the heuristic algorithm on optimization results is examined through repeated experiments. Taking the optimization of TOU electricity pricing in March as an example, 10 groups of experiments are repeated, and the variation of the range of global optimal fitness function with the number of iterations is shown in Fig. 4. In a single experiment, the relationship between the global optimal fitness function and the iteration number is shown in Fig. 5.

According to Fig. 4, although randomness has a great influence on the fitness function, the influence decreases rapidly with the number of iterations. When the number of iterations is more than 160 , the influence of randomness on the optimization results largely disappears. It can be seen from Fig. 5 that although the entry of randomness at the beginning results in a large fitness function, the algorithm converges rapidly. When the number of iterations is close to 20, the global optimal fitness function decreases significantly, while with over 160 iterations, the SAPSO algorithm obtains the global optimal solution.

In addition, the exhaustion method is introduced to verify the global convergence of the optimization results of the SAPSO algorithm. The value range of the TOU price is set as $[0.35,1.2]$, and the interval is divided into 1000 parts on average. The peak, flat, and valley period prices are exhausted respectively, and the fitness function values under different TOU price cases are obtained. The exhaustion method and SAPSO

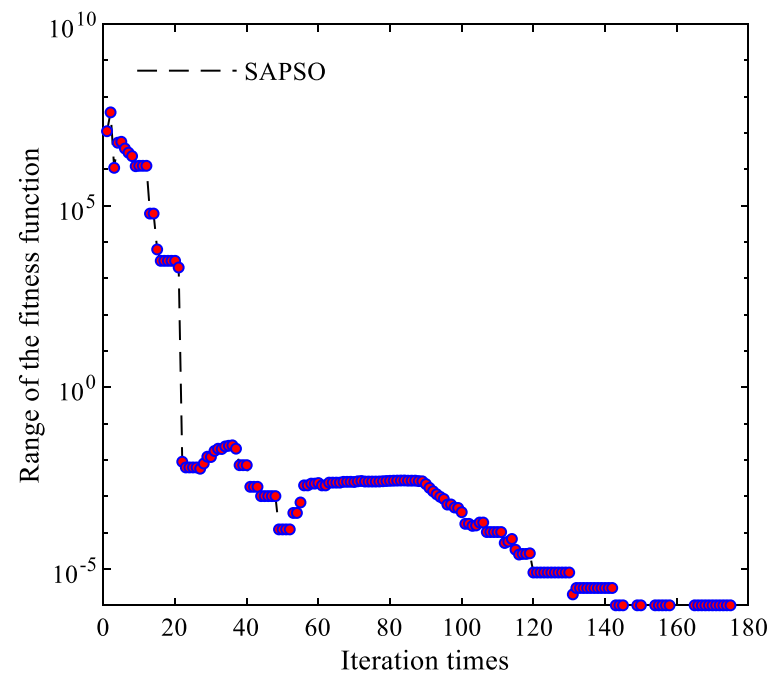

Fig. 4 The relationship between the range of fitness function and the number of iterations

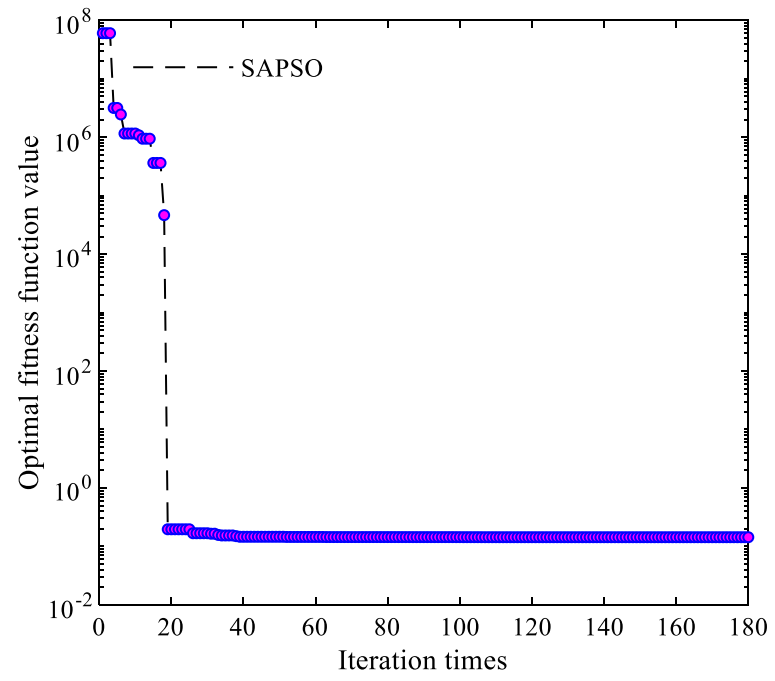

Fig. 5 The relationship between the global optimal fitness function and the number of iterations

algorithm are used to calculate the optimal fitness function for 12 months, as shown in Fig. 6.

It can be seen from Fig. 6 that the optimal fitness function obtained by the SAPSO algorithm is smaller than that of the exhaustion method mentioned above, so the SAPSO algorithm has good global convergence. Because the length of the feasible region is 0.85 , the deviation from the optimal value is less than $8.5 \mathrm{E}-04$, which fills the daily needs. Thus, the SAPSO algorithm proposed in this paper is accurate and effective.

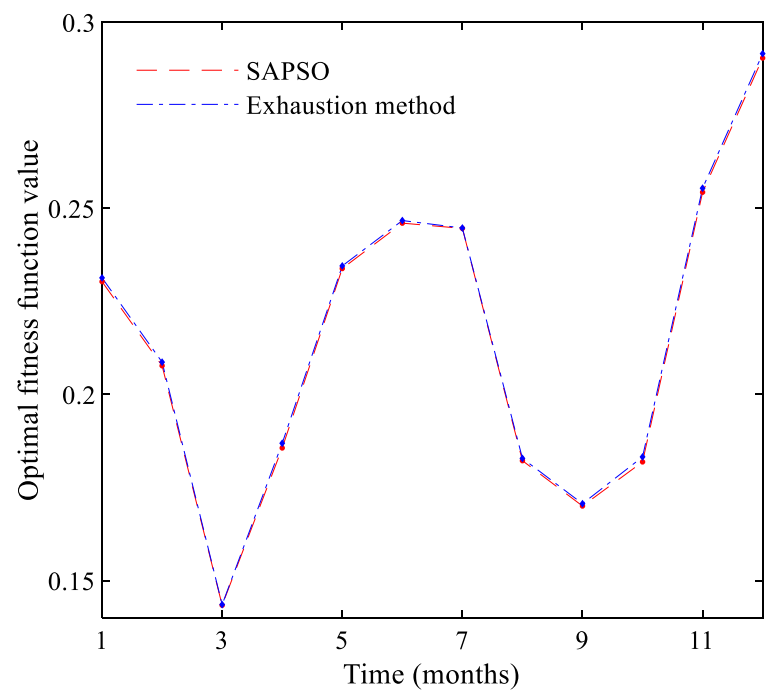

Fig. 6 Comparison between exhaustion method and SAPSO algorithm 


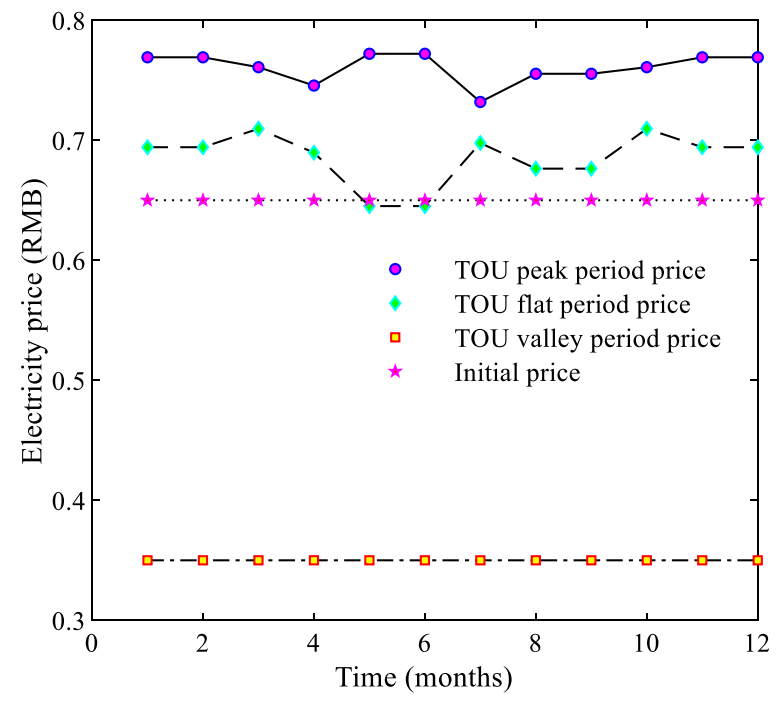

Fig. 7 Optimal results of TOU electricity prices

Table 1 Optimal TOU electricity of different cases

\begin{tabular}{lllll}
\hline Months & Cases & Peak & Flat & Valley \\
\hline January & Case 1 & 0.818 & 0.758 & 0.35 \\
& Case 2 & 0.769 & 0.694 & 0.35 \\
\multirow{2}{*}{ March } & Case 1 & 0.81 & 0.774 & 0.35 \\
& Case 2 & 0.761 & 0.71 & 0.35 \\
\hline
\end{tabular}

\subsection{Effect analysis of TOU price and CPR \\ 5.4.1 Analysis on the effect of TOU price}

The optimal electricity prices for 12 months of the year are shown in Fig. 7. The different peak, flat and valley prices have the effect of reducing the peak load and increasing the valley load. It is noted that the valley price is close to the marginal price, so that the load adjustment effect is optimal.

Based on the initial load of typical days in January and March, two cases are analyzed using the SAPSO algorithm, i.e., Case 1: optimize the TOU price according to the objective functions of the grid side; Case 2: optimize the TOU price by combining the objective functions of the grid side and the user side through (16). The price optimization results of the two cases are shown in Table 1. Based on the hourly load calculation method, the loads at each time corresponding to the price in Table 1 are calculated, as shown in Fig. 8. Table 1 indicates that after considering objective functions of the user side, the peak and flat prices decrease while the valley price remains stable, and thus the users' electricity expenses decrease. It can be concluded from Fig. 8 that after the implementation of the TOU price, the peak load

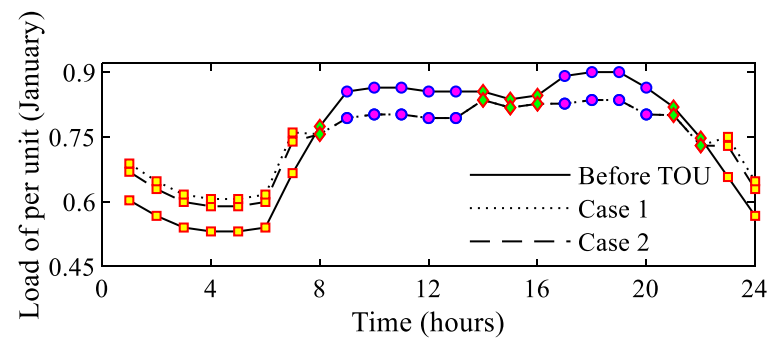

(a)

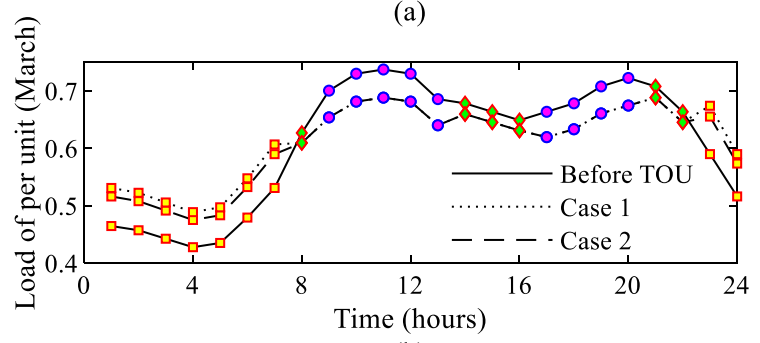

(b)

Fig. 8 Load curves before and after TOU price: a January; b March

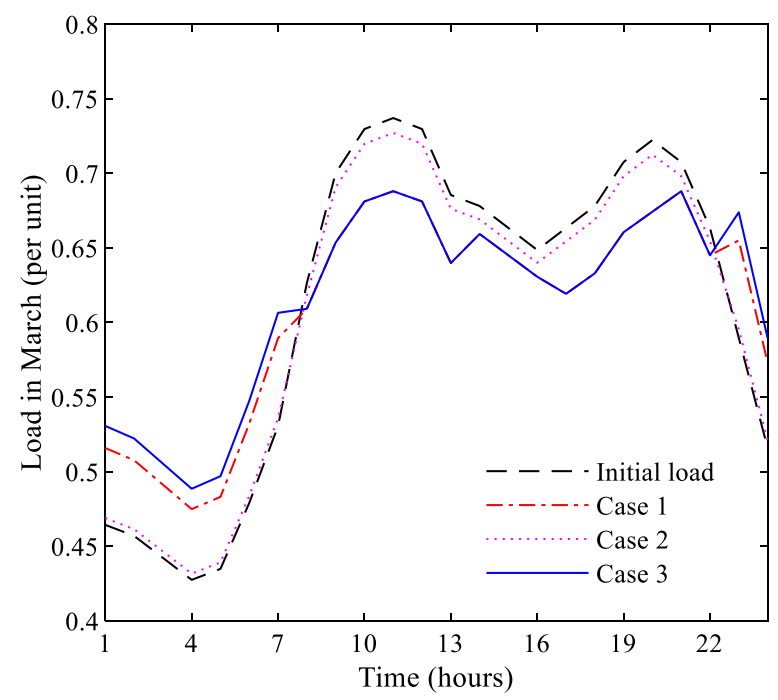

Fig. 9 Load curve of different types of users under TOU price

decreases significantly, while the valley load increases. However, compared with Case 1, the load curve of Case 2 is closer to the initial load, and the power consumption similarity increases accordingly.

The value of $\beta_{i}$ can be used to represent different user characteristics. Three types of users are defined, i.e., Case 1: $\beta_{1}=\beta_{2}=0.5, \beta_{3}=\beta_{4}=-0.3$; Case 2: $\beta_{1}=\beta_{2}=0.01$, $\beta_{3}=\beta_{4}=-0.6$; Case 3: $\beta_{1}=\beta_{2}=0.8, \beta_{3}=\beta_{4}=-0.01$. Taking March as an example, the typical daily load curves of different types of users under TOU pricing are shown in Fig. 9. As can be seen, the load adjustment effect of TOU pricing is different for different types of users. The load 
curve of Case 2 is the closest to the initial curve, while the difference of the peak load and peak-to-valley load in Case 3 are the smallest. In addition, the load curve of Case 1 is the most balanced.

\subsubsection{Effect analysis of CPR}

The load at TOU price is divided into four periods of critical peak, peak, flat, and valley periods. The frequency distribution of each time in critical peak and peak periods of 12 typical days is summarized in Fig. 10. As shown, there are usually two maximum points in the load distribution of a day, and the distribution of the peak period is usually concentrated around 12:00 and 19:00.

Taking the typical days of March and July as examples, the CPR strategy and TC strategy are implemented respectively to adjust the load curves, as shown in Fig. 11. The CPR strategy can reduce the load of the critical peak period and the load difference between the critical peak and peak periods. However, the CPR strategy has little impact on the load in other periods, which ensures user's power consumption similarity.

\subsection{Comparison between TOU pricing and CPR}

Four cases from two aspects of maximum load and reliability are analyzed, including, Case 1: initial load; Case 2: load after TOU price; Case 3: load after CPR strategy; Case 4: load after TC strategy.

\subsubsection{Analysis on load regulation effect of TOU pricing and CPR strategy.}

The differences of the peak load and peak-to-valley load of 12 typical days in four cases are analyzed and compared in Fig. 12. It can be seen that the fluctuation of

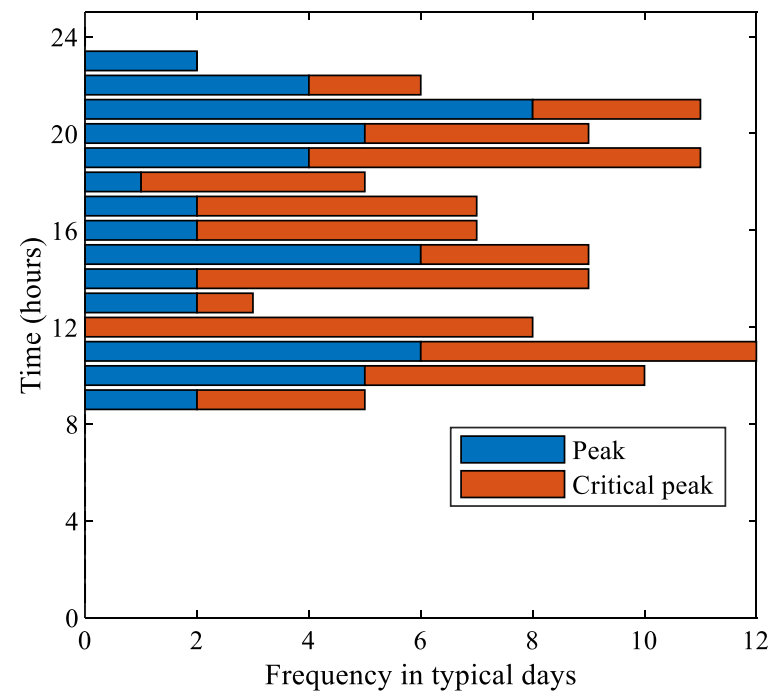

Fig. 10 Distribution of time in different periods

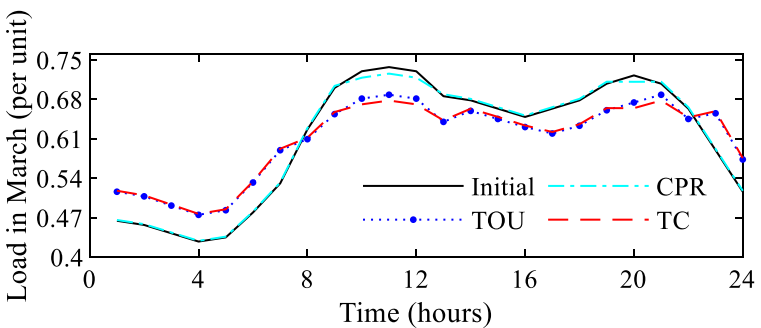

(a)

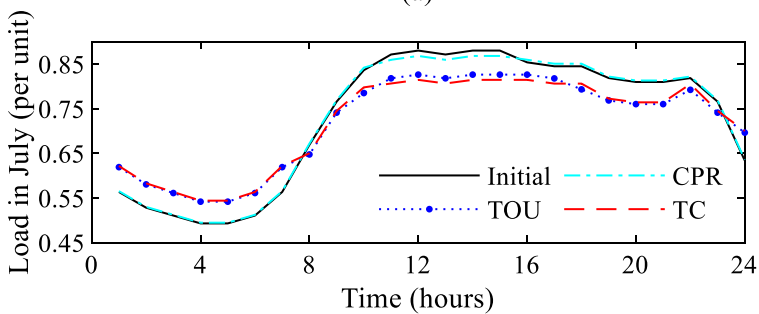

(b)

Fig. 11 Load curves before and after CPR: a March; b July

peak load is much higher than the peak-to-valley load difference under the same regulation mode. Both TOU pricing and CPR strategy can effectively improve load characteristics, especially TOU pricing. The maximum load reduction of CPR under TOU pricing is slightly less than that under the initial price, while the reduction effect of CPR strategy on peak-to-valley load difference is better than that of peak load. Among the cases, Case 4 has the best load characteristics, which proves that the combination of TOU pricing and CPR strategy can maximize the effect of load adjustment.

Based on the 12 typical days, Case 3 and Case 4 are implemented and the critical peak rebates in the two
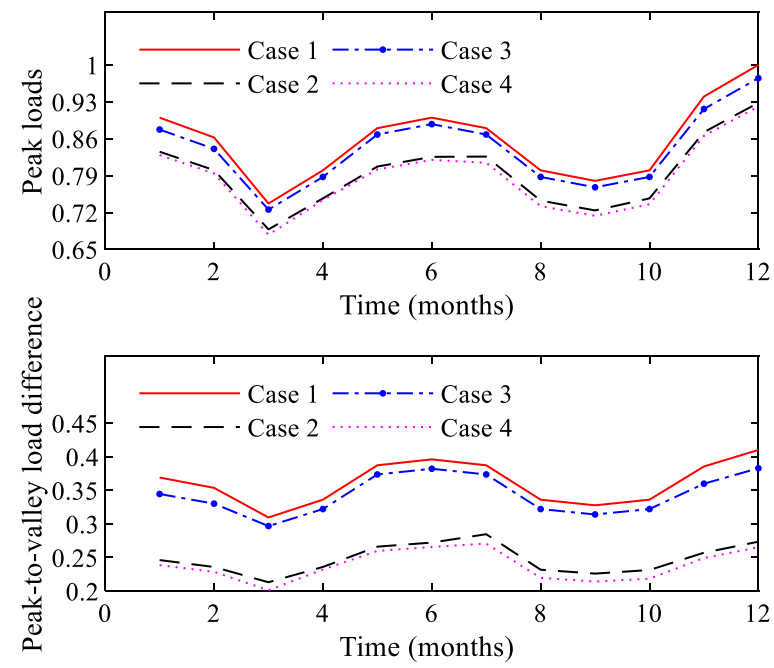

Fig. 12 Load characteristics in different cases 
cases are shown in Fig. 13. The cost of implementing the CPR strategy under TOU pricing is much lower than that of direct implementation. Thus, to reduce the cost of CPR, the CPR strategy should be combined with TOU pricing.

\subsubsection{Reliability comparison before and after load adjustment}

To measure the reliability of the power system in different conditions, loss of load probability (LOLP), loss of load expectation (LOLE) and expected energy not supplied (EENS) are used as the reliability indices:

$$
\begin{aligned}
& \left\{\begin{array}{l}
\text { LOLP }=\sum_{s^{\prime} \in s_{f}} P\left(s^{\prime}\right) \\
\text { LOLE }=\sum_{s^{\prime} \in s_{f}} P\left(s^{\prime}\right) T^{\prime} \\
\text { EENS }=\sum_{s^{\prime} \in s_{f}} \Delta q_{s^{\prime}} P\left(s^{\prime}\right) T^{\prime}
\end{array}\right. \\
& \left\{\begin{array}{l}
S_{f}=\left\{s^{\prime} \sum_{t \in N_{s^{\prime}}} G_{t}<Q_{s^{\prime}}\right. \\
\Delta q_{s^{\prime} \in S_{f}}=Q_{s^{\prime}}-\sum_{t \in N_{s^{\prime}}} G_{t}
\end{array}\right.
\end{aligned}
$$

where $S_{f}$ is the state set of insufficient capacity of the system, $G_{t}$ is the output power of the $t$ th generator, $Q_{s^{\prime}}$ is the load under state $s^{\prime}, T^{\prime}$ is the rated period, $\Delta q_{s^{\prime}}$ is the load reduction caused by insufficient capacity or constrained power generation under state $s^{\prime}$.

The calculated reliability results are shown in Table 2. It is observed that the reliability data of different cases are different. Both TOU pricing and CPR can significantly

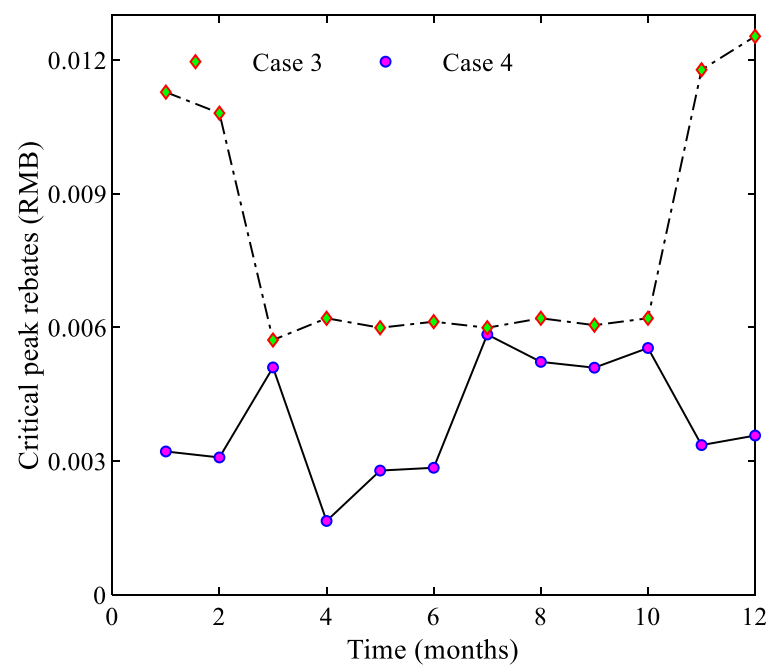

Fig. 13 Critical peak rebates in different cases improve system reliability, but the effect of TOU pricing is more noticeable.

Since the EENS indicators of Case 2 and Case 4 are different, to draw general conclusions, the EENS of the four cases at different peak load levels are analyzed, as shown in Fig. 14. It is observed that with the increase of peak load, the EENS values of the four cases increase, while the values of Case 4 are always the lowest. Thus, the implementation of TC strategy can keep the power system at optimal reliability level under different peak loads.

\section{Conclusions}

This paper studies the optimal demand response decision and reliability analysis based on TOU pricing and CPR strategy. An iterative method of optimal period partition based on fuzzy clustering is proposed, and the typical daily load curve is divided into PFV periods. The optimal TOU price model considering the interests of both the grid and the user is established, and a SAPSO algorithm is proposed to solve the problem. The peak period under TOU pricing is then divided into a critical peak period and a peak period, and a critical peak load adjustment strategy is proposed to reduce the critical peak load proportionally by signing a user agreement. Given the quadratic relationship between power shortage cost and load

Table 2 Analysis of CPR adjustment in different cases

\begin{tabular}{lllll}
\hline Indices & Case 1 & Case 2 & Case 3 & Case 4 \\
\hline LOLP $(\boldsymbol{P})$ & $1.222 \mathrm{E}-04$ & $4.518 \mathrm{E}-05$ & $1.100 \mathrm{E}-04$ & $4.517 \mathrm{E}-05$ \\
LOLE $(\boldsymbol{P})$ & 1.071 & 0.396 & 0.963 & 0.396 \\
EENS $(\boldsymbol{P})$ & 9.734 & 3.301 & 8.811 & 3.139 \\
\hline
\end{tabular}

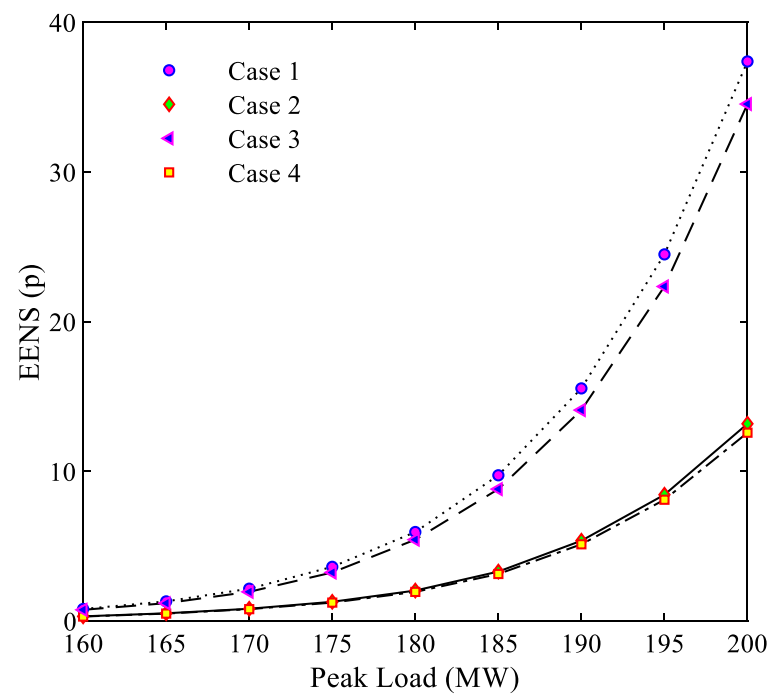

Fig. 14 EENS in different cases 
reduction, the decision-making model of CPR is established to calculate daily critical peak rebate. Finally, the IEEE RBTS reliability data is used to verify and analyze each model.

The following conclusions can be drawn from the case analysis:

1. When using fuzzy clustering and enumeration iteration technology to divide PFV periods, the iteration results tend to be stable when the number of iterations is above 50. It can also be concluded from the period partition results that the lengths of the three periods are similar, and the time composition of each period is relatively fixed.

2. By combining the grid side and user side objective functions to build the TOU price optimization model, it not only reduces peak load and increases valley load, but also ensures user satisfaction and power consumption similarity, as observed from the load curve under TOU pricing.

3. From the established decision-making model of $\mathrm{CPR}$, it is seen that the rebate is positively related to power reduction. To keep compensation costs down, the load reduction should not be too large. The CPR strategy can effectively reduce critical peak load, but the compensation cost of the TC strategy is lower than that of CPR.

4. CPR and TOU pricing can effectively improve system reliability, while the TC strategy can maintain the optimal reliability level under different peak loads.

There are, however, some limitations in the proposed methods in this paper. When the scale of the power system is large and there are many types of users, the selection of a typical day will become an important factor affecting the effect of load adjustment. Therefore, it is necessary to research the selection of typical days in different situations. In addition, in actual scenarios, not all users will be willing to participate in the CPR strategy, even with users' power shortage cost compensated. Therefore, the relationship between the actual participation rate of users and the compensation modes needs to be further explored.

\section{Abbreviations}

TOU:Time-of-use; DSM: Demand side management; CPR: The critical peak rebate; PFV: Peak-flat-valley; SAPSO: Simulated annealing particle swarm optimization; RBTS: Roy Billinton test system; PSO: Particle swarm optimization; SA: Simulated annealing; CPP: Critical peak pricing; TC: The strategy of implementing CPR under TOU price.

\section{Acknowledgements}

Not applicable

\section{Authors' contributions}

Hejun Yang as the corresponding author, building the modeled, designed and tested the proposed strategy and wrote the papers. Xinyu Zhang contributed to write and revise the manuscript and performed some additional changes. Yinghao Ma and Dabo Zhang checked the data in case studies. All authors have read and approved the paper.

\section{Funding}

This work was supported by Fundamental Research Funds for the Central Universities (PA2021 KCPY0053), Anhui Provincial Natural Science Foundation (1908085QE237).

\section{Availability of data and materials}

All data used or analyzed during this study are included in the published article.

\section{Declarations}

\section{Competing interests}

The authors declare that they have no known competing financial interests or personal relationships that could have appeared to influence the work reported in this paper.

Received: 17 December 2020 Accepted: 17 August 2021

Published online: 01 September 2021

\section{References}

1. Jin, H., Li, Z., Sun, H., Guo, Q., \& Wang, B. (2019). Coordination on industrial load control and climate control in manufacturing industry under tou prices. IEEE Transactions on Smart Grid, 10(1), 139-152.

2. Ming, H., Xia, B., Lee, K. Y., Adepoju, A., \& Xie, L. (2020). Prediction and assessment of demand response potential with coupon incentives in highly renewable power systems. Protection and Control of Modern Power Systems, 5, 12.

3. Amroune, M., Bouktir, T., \& Musirin, I. (2019). Power system voltage instability risk mitigation via emergency demand response-based whale optimization algorithm. Protection and Control of Modern Power Systems, 4, 25.

4. Safdarian, A., Fotuhi-Firuzabad, M., \& Lehtonen, M. (2015). A medium-term decision model for discos: Forward contracting and tou pricing. IEEE Transactions on Power Systems, 30(3), 1143-1154.

5. Tran, V. T., Sutanto, D., \& Muttaqi, K. M. (2017). A robust power management strategy entrenched with multi-mode control features for an integrated residential PV and energy storage system to take the advantage of time-of-use electricity pricing. In 2017 IEEE industry applications society annual meeting (pp. 1-8).

6. Burak, K., \& Mouftah, H. T. (2016). Inter-data center network dimensioning under time-of-use pricing. IEEE Transactions on Cloud Computing, 4(4), 402-414.

7. Mohsenian-Rad, A. H., \& Leon-Garcia, A. (2010). Optimal residential load control with price prediction in real-time electricity pricing environments. IEEE Transactions on Smart Grid, 1(2), 120-133.

8. Aalami, H., Yousefi, G. R., \& Moghadam, M. P. (2008). Demand Response model considering EDRP and TOU programs. In Transmission \& distribution conference \& exposition (pp. 1-6).

9. Celebi, E., \& Fuller, J. D. (2012). Time-of-use pricing in electricity markets under different market structures. IEEE Transactions on Power Systems, 27(3), 1170-1181.

10. Yang, P., Tang, G., \& Nehorai, A. (2013). A game-theoretic approach for optimal time-of-use electricity pricing. IEEE Transactions on Power Systems, 28(2), 884-892.

11. Torriti, J. (2012). Price-based demand side management: Assessing the impacts of time-of-use tariffs on residential electricity demand and peak shifting in northern Italy. Energy, 44(1), 576-583.

12. Adika, C. O., \&Wang, L. (2013). Autonomous appliance scheduling based on time of use probabilities and load clustering. In Conference on Ipec (pp. 42-47). 
13. Hu, J., Pan, Y., Li, T., et al. (2021). TW-Co-MFC: Two-level weighted collaborative fuzzy clustering based on maximum entropy for multi-view data. Tsinghua Science and Technology, 26(2), 185-198.

14. Leski, J. M., Czabański, R., Jezewski, M., \& Jezewski, J. (2020). Fuzzy ordered c-means clustering and least angle regression for fuzzy rule-based classifier: Study for imbalanced data. IEEE Transactions on Fuzzy Systems, 28(11), 2799-2813.

15. Shirkhorshidi, A. S., Wah, T.Y., Shirkhorshidi, S. M. R., \& Aghabozorgi, S. (2021). Evolving fuzzy clustering approach: An epoch clustering that enables heuristic postpruning. IEEE Transactions on Fuzzy Systems, 29(3), 560-568.

16. Wei, H., Chen, L., Ruan, K., Li, L., \& Chen, L. (2020). Low-rank tensor regularized fuzzy clustering for multiview data. IEEE Transactions on Fuzzy Systems, 28(12), 3087-3099.

17. Yang, H., Wang, L., Yeyu, Z., et al. (2019). Reliability evaluation of power system considering time of use electricity pricing. IEEE Transactions on Power Systems, 34(3), 1991-2002.

18. Li, Y., Yang, Z., Zhao, D., et al. (2019). Incorporating energy storage and user experience in isolated microgrid dispatch using a multi-objective model. IET Renewable Power Generation, 13(6), 973-981.

19. Yang, H., Wang, L., \& Ma, Y. (2019). Optimal time of use electricity pricing model and its application to electrical distribution system. IEEE Access, 7, 123558-123568.

20. Liu, F., Yang, L., Xiao, S., \& Zhang, D. (2010). Application of RAGA based on SA in calculating critical water depth of open channel. In 6th international conference on natural computation (pp. 2296-2300).

21. Wolak, F. A. (2007). Residential customer response to real-time pricing: The Anaheim critical peak pricing experiment. In Center for the Study of Energy Markets (CSEM) Working Paper Series (pp. 1-5).
22. Li, Y., Gao, W., Ruan, Y., et al. (2018). Demand response of customers in Kitakyushu smart community project to critical peak pricing of electricity. Energy \& Buildings, 168, 251-260.

23. Park, S. C., Jin, Y. G., Song, H. Y., \& Yoon, Y. T. (2015). Designing a critical peak pricing scheme for the profit maximization objective considering price responsiveness of customers. Energy, 83(a1), 521-531.

24. Federal Energy Regulatory Commission. (2006). Assessment of demand response and advanced metering: staff report, (pp. 22-39). https://www. ferc.gov/legal/staff-reports/demand-response.pdf.

25. Fahrioglu, M., \& Alvarado, F. L. (2001). Using utility information to calibrate customer demand management behavior models. IEEE Transactions on Power Systems, 16(2), 317-322.

26. Fahrioglu, M., \& Alvarado, F. L. (2000). Designing incentive compatible contracts for effective demand management. IEEE Transactions on Power Systems, 15(4), 1255-1260.

27. Jiang, H., Liu, B., Wang, Y., \& Zheng, S. (2014). Multiobjective tou pricing optimization based on nsga2. Journal of Applied Mathematics, 2014(2), $1-8$.

28. Parsopoulos, K. E., \& Vrahatis, M. N. (2002). Particle swarm optimization method for constrained optimization problem. Frontiers in Artificial Intelligence, 76(1), 214-220.

29. Billinton, R., Kumar, S., Chowdhury, N., Chu, K., Debnath, K., Goel, L., et al. (1989). A reliability test system for educational purposes-basic data. IEEE Transactions on Power Systems, 4(3), 1238-1244.

30. Subcommittee, P. M. (1979). IEEE reliability test system. IEEE Transactions on Power Apparatus and Systems PAS, 98(6), 2047-2054.

\section{Submit your manuscript to a SpringerOpen ${ }^{\circ}$ journal and benefit from:}

- Convenient online submission

- Rigorous peer review

- Open access: articles freely available online

- High visibility within the field

- Retaining the copyright to your article

Submit your next manuscript at $\boldsymbol{\nabla}$ springeropen.com 